Pacific

Journal of

Mathematics

EXPONENTS OF A MEROMORPHIC CONNECTION ON A COMPACT RIEMANN SURFACE

EDUARDO COREL

Volume $242 \quad$ No. 2

October 2009 


\title{
EXPONENTS OF A MEROMORPHIC CONNECTION ON A COMPACT RIEMANN SURFACE
}

\author{
EDUARDO COREL
}

\begin{abstract}
We give a general definition of the exponents of a meromorphic connection $\nabla$ on a holomorphic vector bundle $\mathscr{E}$ of rank $n$ over a compact Riemann surface $X$. We prove that they can be computed as invariants of a vector bundle $\mathscr{E}_{L}$ canonically attached to $\mathscr{E}$, which we construct and call the Levelt bundle of $\mathscr{E}$, and whose degree (equal to the sum of the exponents) we estimate by upper and lower bounds (Fuchs' relations). We use this definition to construct, for every linear differential equation on a compact Riemann surface (with regular or irregular singularities), the companion bundle of the equation, a vector bundle endowed with a meromorphic connection that is equivalent to the given equation and has precisely the same singularities and the same set of exponents.
\end{abstract}

\section{Introduction}

Exponents of differential equations are complex numbers that describe the behavior of solutions in a neighborhood of a singular point. Consider a fuchsian differential equation with coefficients in $\mathbb{C}(z)$ :

$$
y^{(n)}+a_{1} y^{(n-1)}+\cdots+a_{n} y=0 .
$$

Its exponents $\left(e_{i}^{s}\right)_{1 \leqslant i \leqslant n}$ for $s \in \mathbb{P}^{1}(\mathbb{C})$, defined by Fuchs [1866], obey the celebrated Fuchs relation

$$
\sum_{s \in \mathbb{P}^{1}(\mathbb{C})} \sum_{i=1}^{n}\left(e_{i}^{s}-(i-1)\right)=-n(n-1) .
$$

This relation, and several generalizations — on a Riemann surface in [Saito 1958] and for irregular singularities in [Bertrand and Beukers 1985; Chudnovsky and Chudnovsky 1984], for example — have been used in arithmetic, following ideas by C. L. Siegel, to give transcendence estimates for values of special functions

MSC2000: primary 14F05, 34A30, 34M45; secondary 34M50, 12H05, 30F30.

Keywords: meromorphic connections, exponents, Fuchs' relations, Levelt bundle.

This work was done in part while the author was supported by the French CNRS during a postdoctoral stay at the Laboratoire Statistique et Génome, Evry (France). 
on the basis of differential equations that they satisfy. Basically, differential equations and differential systems are equivalent, and both could be used for the same purpose. However, the theory of exponents for systems has lagged behind some 80 years, probably due to the absence of such a nice characterization of a regular singular point as provided by Fuchs' theorem for equations.

The counterexample given by A. A. Bolibrukh [1990b] to the strong RiemannHilbert problem (that is, with simple poles) is based on a Fuchs relation argument for differential systems, and has shown yet another application for this notion. Indeed, if

$$
\frac{d X}{d z}=A X, \quad \text { where } A \in \mathrm{M}_{n}(\mathbb{C}(z))
$$

is a meromorphic differential system with regular singularities on $\mathbb{P}^{1}(\mathbb{C})$, Bolibrukh proves that its exponents $\mu_{1}^{s}, \ldots, \mu_{n}^{s}$, defined by Levelt [1961], satisfy the relation $\sum_{s \in \mathbb{P}^{1}(\mathbb{C})} \sum_{i=1}^{n} \mu_{i}^{s} \leqslant 0$, and the equality is achieved exactly when the system has only simple poles; such systems are called Fuchsian.

Although both categories are usually treated as strictly equivalent, the sum of exponents for equations is a positive integer, while the sum of exponents for systems is negative. Hence, there cannot exist a differential system that is globally equivalent to a differential equation, in that it has the same exponents everywhere. In the regular case, such equivalent constructions (by way of a companion matrix) are always possible locally, as is well known. To perform this globally, one must glue these local data together; therefore we must introduce the framework of holomorphic vector bundles equipped with a meromorphic connection.

The first contribution of this paper is to bridge the gap between the two notions of exponents by constructing in a canonical way such a vector bundle (the companion bundle of the differential equation), which gives the correct local exponents at every singularity. In case the equation is fuchsian, the vector bundle that we get has been constructed by M. F. Singer and M. van der Put [2003], albeit from another perspective. In the irregular case, the exponents of the companion bundle are equal to the exponents $\left(\lambda_{i}^{s}\right)_{1 \leqslant i \leqslant n}$ for $s \in \mathbb{P}^{1}(\mathbb{C})$, defined by D. Bertrand and G. Laumon [1985] as a generalization of Fuchs' exponents, for the arithmetical purposes explained above. In this last case, a ramification of the variable is usually required. For the construction of the companion bundle, it is thus necessary to replace the Riemann sphere with a compact covering $\pi: X \rightarrow \mathbb{P}^{1}(\mathbb{C})$, ramified over the singular points of the equation.

This requires therefore a precise definition of the exponents $\mu_{1}^{\mathscr{E}, s}, \ldots, \mu_{n}^{\mathscr{E}, s}$ at any point $s \in X$ for a vector bundle $\mathscr{E}$ endowed with a meromorphic connection $\nabla$ on a Riemann surface $X$. Our algebraic Definition 3, which extends the notion of exponents defined by Levelt for the regular case, involves the construction of a 
special "well-behaved" Levelt bundle $\mathscr{E}_{L}$ of the bundle $\mathscr{E}$; see Theorem 1 . This part of the article builds on local results from [Corel 2004], in which we defined the exponents of the linear differential system (1) at an irregular singularity $s \in \mathbb{P}^{1}(\mathbb{C})$.

First, we prove that these exponents are defined in a coordinate-invariant way (unlike for instance the generalized exponents defined by Van Hoeij [1997], whose Laurent polynomial part depends on the chosen local coordinate); consequently, we find that the Bertrand-Laumon exponents are well defined on a Riemann surface.

Second, we derive in Theorem 4 new Fuchs-like estimates for the sum of the exponents of a general meromorphic connection on a holomorphic bundle. Arithmetic applications therefore do not need to be restricted to actual solutions of differential equations, but can be used with horizontal sections of arbitrary meromorphic connections on any compact Riemann surface.

Finally, the definition of the Levelt bundle can shed some light on the RiemannHilbert problem and some of its generalizations. P. Deligne [1970] showed how to extend a holomorphic vector bundle $\mathscr{E}$, defined over the complement of a divisor $\mathscr{D}$ and endowed with a holomorphic connection $\nabla$ having regular singularities on $\mathscr{D}$, into a logarithmic connection $(\bar{E}, \bar{\nabla})$ that has singularities on the divisor and is uniquely determined by a section of the natural projection $\mathbb{C} \rightarrow \mathbb{C} / \mathbb{Z}$. In this way, we get all logarithmic extensions of $\mathscr{E}$ with nonresonant residue; these are the Deligne lattices. This construction is sufficient to solve positively the weak Riemann-Hilbert problem (that is, the one with regular singularities). The strong Riemann-Hilbert problem however asks for a logarithmic bundle (with the prescribed monodromy) that is also trivial. Bolibrukh's counterexample requires therefore the knowledge of all the logarithmic extensions of a regular connection in order to prove that none is trivial. The Levelt bundles, in the regular case, correspond precisely to the missing logarithmic extensions, those for which we allow the residue to be resonant.

In the irregular case, the Levelt bundle is, roughly, the "closest" bundle with the property of being locally split in isotypical components, themselves logarithmic modulo an irregular part (we call this property "log-split"). Like in the regular case, the Levelt bundle happens to be the extension to the resonant case of a classical construction, here Malgrange's [1996] canonical bundle, which extends Deligne's to the irregular case. It is also a natural extension of the notion of "logarithmic bundle" as it is likewise characterized by a Fuchs relation (see Theorem 3)

$$
\mathscr{E} \text { is log-split if and only if } \sum_{s \in X} \sum_{i=1}^{n} \mu_{i}^{\mathscr{E}, s}=\operatorname{deg} \mathscr{E} .
$$

In the formulation of Bolibrukh, Malek and Mitschi [Bolibruch et al. 2006], the generalized Riemann-Hilbert problem asks for a trivial bundle with minimal Poincaré ranks with respect to the unique connection satisfying some prescribed 
"generalized monodromy data". As one expects, the reducibility of the monodromy is involved in the solubility, but more unexpectedly, ramification (or rather its absence) also is. Van der Put and Saito [2009] have already provided a counterexample to this version of the problem. However, it is not clear whether a bundle with minimal Poincaré ranks provides the most natural generalization of the logarithmic bundles required in the regular case. Indeed, a bundle fails to be logarithmic when the connection exhibits nilpotency (appearing under the form of an irregular part whose leading term is nilpotent) that can be suppressed by meromorphic gauge transforms. Requiring minimal Poincaré rank in the irregular case does not prevent the regular part of the connection from having a residual nilpotency hidden under the terms of the irregular part (this can result, for example, in a nondiagonalizable leading term). In terms of the Babbitt-Varadarajan decomposition of the connection into a regular connection and an irregular part [Babbitt and Varadarajan 1983; Corel 2004], the extension of the Fuchs relation for Fuchsian systems as formula (2) suggests that the equivalent of "logarithmic" in the irregular case requires minimal (that is, zero) Poincaré rank for the regular part, and diagonalizability for the irregular part of the connection, namely that the bundle be log-split. At any rate, it would be interesting to know if, in the unramified case at least, the variant of the generalized Riemann-Hilbert problem asking for a trivial log-split bundle admits a solution. On the other hand, the tools introduced here also apply for the minimum Poincaré rank case (Proposition 1), so interesting insights can be reasonably expected, although they would require more work, since the corresponding bundle has no direct characterization by exponents.

In Section 1, we prove that the exponents of a meromorphic connection on a holomorphic vector bundle can be defined by a global object, the Levelt bundle; we do this basically by gluing the local Levelt lattices constructed in [Corel 2004]. For these exponents, we establish new estimates based on the local Jordan structure of the residual maps of the connection. In Section 2, we construct the companion bundle of a differential equation, and in Section 3, we establish the global estimates.

\section{The Levelt bundle and the exponents}

Let $X$ be a compact Riemann surface and $\mathscr{D}=\sum_{x \in X} m_{x} x$ be a positive divisor on $X$ with (finite) support $|\mathscr{D}|=\left\{x \in X \mid m_{x} \neq 0\right\}$. Let $O_{\mathscr{D}}$ be the sheaf of meromorphic functions on $X$ whose poles have order bounded by $m_{x}$ at $x \in X$. For a locally free sheaf $\mathscr{E}$ of $\mathscr{O}_{X}$-modules (subsequently called a vector bundle) of finite rank on $X$, let $\mathscr{E} \mathscr{D}=\mathscr{E} \otimes_{\mathscr{O}_{X}} \mathscr{O}_{\mathscr{D}}$. Let $\nabla: \mathscr{E} \rightarrow \mathscr{E} \otimes_{\mathscr{O}_{X}} \Omega_{\mathscr{D}}$ be a meromorphic connection with singular divisor $\mathscr{D}$ on a vector bundle $\mathscr{E}$ of rank $n$. In the sequel, we will always assume that $\mathscr{D}$ is the smallest possible. Sometimes for simplicity we'll just say "connection" for the pair $(\mathscr{E}, \nabla)$. The Poincaré rank of $\nabla$ at $x \in X$ is the integer 
$\mathfrak{p}_{x}(\nabla)=\max \left(0, m_{x}-1\right)$. If $\mathfrak{p}_{x}(\nabla)=0$, the sheaf $\mathscr{E}$ is said to be logarithmic with respect to $\nabla$ at $x$. Let $\mathscr{S}=|\mathscr{D}|$ be the singular, and $\mathscr{S}_{\log }=\left\{x \in \mathscr{Y} \mid \mathfrak{p}_{x}(\nabla)=0\right\}$ the logarithmic singular sets of $\nabla$. If $\mathscr{Y}_{\log } \neq \varnothing$, then one can define the residue map Res $\nabla \in \mathscr{E} n d\left(\mathscr{E} / \mathscr{E}_{-\left[\mathscr{S}_{\log }\right]}\right)$, where $\left[\left\{x_{1}, \ldots, x_{m}\right\}\right]=x_{1}+\cdots+x_{m}$. If $x \in \mathscr{Y} \backslash \mathscr{Y}_{\log }$ is not logarithmic, the residue of $z^{\mathfrak{p}_{x}(\nabla)} \nabla$ for any local coordinate $z$ induces the well-defined polar map $P M \nabla \in \mathbb{P} \mathscr{E} n d\left(\mathscr{E} / \mathscr{E}_{-}[9]\right)$ of $\nabla$ over $\mathscr{E}$. For more details on these notions, see [Sabbah 2002].

1.1. Local maximal lattices. Let $x \in \mathscr{Y}$. Let $\widehat{O}=\widehat{\mathrm{O}_{X, x}}$ be the formal completion of the ring $\mathrm{O}_{X, x}$ endowed with its discrete valuation $v$ with respect to its maximal ideal $\mathfrak{m}$, and let $K$ be the fraction field of $\mathcal{O}$. The $\mathcal{O}$-module $\Lambda=\mathscr{E}_{x} \otimes_{\mathscr{O}_{X, x}} \mathcal{O}$ is a lattice in the $K$-vector space $V=\Lambda \otimes_{0} K$, on which it induces an additive valuation $v_{\Lambda}(x)=\max \left\{k \in \mathbb{Z} \mid x \in \mathfrak{m}^{k} \Lambda\right\}$ for $x \in V$. Let $\Omega=\Omega_{X, x} \otimes_{\mathcal{O}_{X, x}} K$ and $\Omega(k)=\{\omega \in \Omega \mid v(\omega) \geqslant-k\}$. The germ of connection

$$
\nabla_{x}: \mathscr{E}_{x} \rightarrow \mathscr{E}_{x} \otimes_{\mathscr{O}_{X, x}} \Omega_{\mathscr{D}, x}
$$

extends as the map $\nabla=\nabla_{x} \otimes \mathrm{id}_{K}+\mathrm{id}_{V} \otimes d: V \rightarrow V \otimes_{K} \Omega$. The triple $(V, \Lambda, \nabla)$ is called the (formal) local model of $(\mathscr{E}, \nabla)$ at $x$.

Let $z$ be a uniformizing parameter of $K$, and consider the derivation $\theta=z \frac{d}{d z}$. The Poincaré rank $\mathfrak{p}$ of $\nabla$ at $x$ satisfies $\mathfrak{p}=-v_{\Lambda}\left(\nabla_{\theta}(\Lambda)+\mathfrak{m} \Lambda\right)$, where $v_{\Lambda}(M)=$ $\inf _{x \in M} v_{\Lambda}(x)$. The map $\delta_{\Lambda}$ induced by $z^{\mathfrak{p}} \nabla_{\theta}$ on $\bar{\Lambda}=\Lambda / \mathfrak{m} \Lambda$ is a representative of the germ $(P M \nabla)_{x}$. If $\mathfrak{p}=0$, put $\delta_{\Lambda}=0$. For any integer $\ell>0$, we have $z^{\ell \mathfrak{p}} \nabla_{\theta}^{\ell}(\Lambda)=\left(z^{\mathfrak{p}} \nabla_{\theta}\right)^{\ell} \bmod \mathfrak{m} \Lambda$. The map induced by either on $\bar{\Lambda}=\Lambda / \mathfrak{m} \Lambda$ is equal to $\delta_{\Lambda}^{\ell}$. Let $\mathscr{K}=\left(k_{1}, \ldots, k_{n}\right)$ be the (increasing) sequence of elementary divisors of a lattice $M \subset \Lambda$, and let $[\Lambda: M]=\sum_{i=1}^{n}\left(k_{i}-k_{1}\right)$ be its index with respect to $\Lambda$. A lattice $M$ in $V$ induces a natural flag in $\bar{\Lambda}$ as follows. Let

$$
\mathfrak{m} \Lambda \subset M_{k}=\left(z^{-k} M \cap \Lambda\right)+\mathfrak{m} \Lambda \subset \Lambda \quad \text { for } k \in \mathbb{Z} .
$$

The images $\bar{M}_{k}$ in the quotient space $\bar{\Lambda}$ induce a flag of $\mathbb{C}$-vector spaces

$$
F_{\Lambda}(M): 0=\mathscr{F}_{0} \subsetneq \mathscr{F}_{1} \subsetneq \cdots \subsetneq \mathscr{F}_{s}=\bar{\Lambda},
$$

where $\mathscr{F}_{i}=\bar{M}_{\mathbf{k}_{i}}$; here $\mathbf{k}_{i}$ is the $i$-th distinct elementary divisor of $M$.

Let $m(\nabla)$ be the order of singularity of $\nabla$, that is, the minimum of the Poincare ranks over all lattices. For any $\mathfrak{q} \geqslant m(\nabla)$, there exists a largest lattice of $\Lambda_{\mathfrak{q}} \subset \Lambda$ having Poincaré rank $\leqslant \mathfrak{q}$; see [Corel 2004, Corollary 2.2].

Proposition 1. Let $\Lambda$ be a lattice in $V$ with Poincaré rank p. Let $\delta_{i}=\operatorname{rk}\left(\delta_{\Lambda}\right)^{i}$ and $\Delta_{i}=\delta_{i}-\delta_{i+1}$ for $i \geqslant 0$. For $m(\nabla) \leqslant \mathfrak{q} \leqslant \mathfrak{p}$, the maximal lattice $\Lambda_{\mathfrak{q}} \subset \Lambda$ satisfies

$$
d \sum_{i \geqslant 1} \delta_{i} \leqslant\left[\Lambda: \Lambda_{\mathfrak{q}}\right] \leqslant \frac{1}{2} n(n-1) d-\frac{1}{2} \sum_{i \geqslant 0} \Delta_{i}\left(\Delta_{i}-1\right), \quad \text { where } d=\mathfrak{p}-\mathfrak{q} .
$$


Let $\Lambda$ and $M$ be lattices in $V$, and let $\mathfrak{p}_{\Lambda}$ and $\mathfrak{p}_{M}$ be their respective Poincaré ranks with respect to the connection $\nabla$.

Lemma 1. For $k \in \mathbb{Z}$, one has $\delta_{\Lambda}\left(\bar{M}_{k}\right) \subset \bar{M}_{\ell}$, where $\ell=k+\left(\mathfrak{p}_{M}-\mathfrak{p}_{\Lambda}\right)$.

Proof. Put $p=\mathfrak{p}_{\Lambda}$, let $m \in M$ such that $z^{-k} m \in \Lambda$ holds, and let $\lambda \in \Lambda$. Then,

$$
z^{p} \nabla_{\theta}\left(z^{-k} m+z \lambda\right)=-k z^{p-k} m+z^{-k} z^{p} \nabla_{\theta}(m)+z\left(z^{p} \nabla_{\theta}(\lambda)+z^{p} \lambda\right) .
$$

By assumption, $z^{p-k} m \in z^{p-k} M \cap z^{p} \Lambda$ holds, and so does $z\left(z^{p} \nabla_{\theta}(\lambda)+z^{p} \lambda\right) \in \mathfrak{m} \Lambda$. By definition of Poincaré rank, we have $z^{p} \nabla_{\theta}(m)=z^{p-\mathfrak{p}_{M}}\left(z^{\mathfrak{p}_{M}} \nabla_{\theta}(m)\right) \in z^{p-\mathfrak{p}_{M}} M$, and, since $m \in z^{k} \Lambda$, we then get $z^{p} \nabla_{\theta}(m) \in z^{k} \Lambda$. Accordingly, we find that $z^{-k} z^{p} \nabla_{\theta}(m) \in z^{-k}\left(z^{p-\mathfrak{p}_{M}} M \cap z^{k} \Lambda\right)=z^{-\left(k+\mathfrak{p}_{M}-p\right)} M \cap \Lambda$. Consequently, we have $z^{p} \nabla_{\theta}\left(z^{-k} m+z \lambda\right) \in\left(z^{-\left(k+\mathfrak{p}_{M}-p\right)} M \cap \Lambda\right)+\mathfrak{m} \Lambda=M_{k+\left(\mathfrak{p}_{M}-p\right)}$, and the lemma is established by taking the quotient by $\mathfrak{m} \Lambda$.

Corollary 1. Let $M$ be a lattice having Poincaré rank $\mathfrak{p}_{M}<\mathfrak{p}_{\Lambda}$ and elementary divisors $\left(k_{1}, \ldots, k_{n}\right)$ with respect to $\Lambda$. Let $F_{\Lambda}(M): 0=\mathscr{F}_{0} \subsetneq \mathscr{F}_{1} \subsetneq \ldots \subsetneq \mathscr{F}_{s}=\bar{\Lambda}$ be the flag induced by $M$.

(i) The flag $F_{\Lambda}(M)$ satisfies $\delta_{\Lambda}\left(\mathscr{F}_{i}\right) \subset \mathscr{F}_{i-1}$ for all $1 \leqslant i \leqslant s$.

(ii) If $k_{i+1}-k_{i}<\mathfrak{p}_{\Lambda}-\mathfrak{p}_{M}$, the graduated map $\operatorname{gr}_{i+1}^{\mathscr{F}_{F}}\left(\delta_{\Lambda}\right): \mathscr{F}_{i+1} / \mathscr{F}_{i} \rightarrow \mathscr{F}_{i} / \mathscr{F}_{i-1}$ is equal to 0 .

(iii) For $\ell>0$, let $t=\max \left\{1 \leqslant i \leqslant n \mid k_{i}-k_{1}<\ell\left(\mathfrak{p}_{\Lambda}-\mathfrak{p}_{M}\right)\right\}$. Then $\bar{M}_{t} \subset \operatorname{ker} \delta_{\Lambda}^{\ell}$.

Proof. The three assertions are consequences of Lemma 1. Indeed, if $\mathscr{F}_{i}=\bar{M}_{k_{i}}$, then $\delta_{\Lambda}\left(\mathscr{F}_{i}\right) \subset \bar{M}_{k_{i}+\mathfrak{p}_{M}-\mathfrak{p}_{\Lambda}} \subset \bar{M}_{k_{i}-1}=\bar{M}_{k_{i-1}}=\mathscr{F}_{i-1}$. The second is established by remarking that if $k_{i+1}-k_{i}<\mathfrak{p}_{\Lambda}-\mathfrak{p}_{M}$, then $k_{i+1}+\mathfrak{p}_{M}-\mathfrak{p}_{\Lambda}<k_{i}$, and therefore $k_{i+1}+\mathfrak{p}_{M}-\mathfrak{p}_{\Lambda} \leqslant k_{i}-1$. Thus $\delta_{\Lambda}\left(\mathscr{F}_{i+1}\right) \subset \bar{M}_{k_{i}-1}=\mathscr{F}_{i-1}$. Finally, one has $\delta_{\Lambda}^{\ell}\left(\bar{M}_{t}\right) \subset \bar{M}_{k_{t}+\ell\left(\mathfrak{p}_{M}-\mathfrak{p}_{\Lambda}\right)} \subset \bar{M}_{k_{1}-1}=0$, since $k_{t}-\ell\left(\mathfrak{p}_{M}-\mathfrak{p}_{\Lambda}\right)<k_{1}$ according to the definition of $t$; this establishes the third result.

Let $\mathscr{K}=\left(k_{1}, \ldots, k_{n}\right)$ be the elementary divisors of $\Lambda_{\mathfrak{q}}$ in $\Lambda$, and let $\mathscr{F}$ be the flag induced by $\Lambda_{\mathfrak{q}}$ in $\Lambda / \mathfrak{m} \Lambda$. Recall (see [Corel 2004, Proposition 4.2]) that $k_{1}=0$ and $\max _{i=1, \ldots, n-1} k_{i+i}-k_{i} \leqslant \mathfrak{p}-\mathfrak{q} \leqslant k_{n}$. In this case, the converse of Corollary 1(ii) holds.

Lemma 2. With the previous notations, $k_{i+1}-k_{i}=\mathfrak{p}-\mathfrak{q}$ if and only if $\operatorname{gr}_{i+1}^{\mathscr{F}}\left(\delta_{\Lambda}\right) \neq 0$.

Proof. Let (e) be a basis of $\Lambda$ and $(\varepsilon)$ a basis of $\Lambda_{\mathfrak{q}}$ such that the matrix of the basis change from $(e)$ to $(\varepsilon)$ is $z^{K}$ where $K=\operatorname{diag}\left(k_{1} I_{n_{1}}, \ldots, k_{s} I_{n_{s}}\right)$, by considering the elementary divisors with multiplicities. Recall that $n_{i}=\operatorname{dim} \mathscr{F}_{i}-\operatorname{dim} \mathscr{F}_{i-1}$. This means to consider the sequence $t_{1}<\cdots<t_{s}$ such that $n_{j}=t_{j}-t_{j-1}+1$ and

$$
0=k_{1}=\cdots=k_{t_{1}}<k_{t_{1}+1}=\cdots=k_{t_{2}}<\cdots<k_{t_{s-1}+1}=\cdots=k_{t_{s}}=k_{n} .
$$


Let $A=\operatorname{Mat}\left(\nabla_{\theta},(e)\right)=A_{-\mathfrak{p}} z^{-\mathfrak{p}}+\cdots$. The matrix $A_{-\mathfrak{p}}$ has the block-triangular form

$$
A_{-\mathfrak{p}}=\left(\begin{array}{llll}
0_{n_{1}} & \mathscr{A}_{12} & \cdots & \mathscr{A}_{1 s} \\
0 & 0_{n_{2}} & \cdots & \mathscr{A}_{2 s} \\
\vdots & & \ddots & \vdots \\
0 & 0 & \cdots & 0_{n_{s}}
\end{array}\right)
$$

Let $\ell \in\{1, \ldots, n-1\}$ such that $k_{\ell}>0$, and consider the $\tilde{\mathscr{K}}=\left(\tilde{k}_{1}, \ldots, \tilde{k}_{n}\right)$, where $\tilde{k}_{i}=k_{i}$ for $i \leqslant \ell$ and $\tilde{k}_{j}=k_{j}-1$ for $j \geqslant \ell+1$. Since $\Lambda_{\mathfrak{q}}$ is maximal, there exists $\left(i_{0}, j_{0}\right)$ such that $v\left(A_{i_{0}, j_{0}}\right)+\tilde{k}_{j_{0}}-\tilde{k}_{i_{0}}<-\mathfrak{q}$. We have

$$
\tilde{k}_{j}-\tilde{k}_{i}= \begin{cases}k_{j}-k_{i} & \text { if } i \leqslant \ell \text { and } j \leqslant \ell, \text { or } i \geqslant \ell+1 \text { and } j \geqslant \ell+1, \\ k_{j}-k_{i}+1 & \text { if } i \geqslant \ell+1 \text { and } j \leqslant \ell, \\ k_{j}-k_{i}-1 & \text { if } i \leqslant \ell \quad \text { and } j \geqslant \ell+1 .\end{cases}
$$

Thus, we get $i_{0} \leqslant \ell<\ell+1 \leqslant j_{0}$, and accordingly $v\left(A_{i_{0}, j_{0}}\right)+k_{j_{0}}-k_{i_{0}}=-\mathfrak{q}$. Assume that $k_{\ell+1}-k_{\ell}=\mathfrak{p}-\mathfrak{q}$. Since $k_{j_{0}}-k_{i_{0}} \geqslant k_{\ell+1}-k_{\ell}$, we have

$$
-\mathfrak{p} \leqslant v\left(A_{i_{0}, j_{0}}\right) \leqslant-k_{j_{0}}+k_{i_{0}}-\mathfrak{q} \leqslant-\mathfrak{p}
$$

hence $v\left(A_{i_{0}, j_{0}}\right)=-\mathfrak{p}$. Therefore $\left(A_{-\mathfrak{p}}\right)_{i_{0}, j_{0}} \neq 0$ with $k_{i_{0}}=k_{\ell}$ and $k_{j_{0}}=k_{\ell+1}$. Let $u$ be the index such that $k_{\ell}=k_{t_{u}}$. Then, in the block structure of $A_{-\mathfrak{p}}$ we have rk $\mathscr{A}_{u, u+1} \geqslant 1$. This implies that $\mathscr{A}_{u, u+1} \neq 0$ if and only if $k_{t_{u+1}}-k_{t_{u}}=\mathfrak{p}-\mathfrak{q}$. But $\mathscr{A}_{u, u+1}$ is nothing but the matrix of the graduated map $\operatorname{gr}_{u+1}^{\mathscr{F}}\left(\delta_{\Lambda}\right)$.

Let $\ell \geqslant 0$ be an integer. Let $a_{\ell}=\max \left\{1 \leqslant i \leqslant n \mid k_{i}<\ell(\mathfrak{p}-\mathfrak{q})\right\}$ and $a_{0}=0$, and let

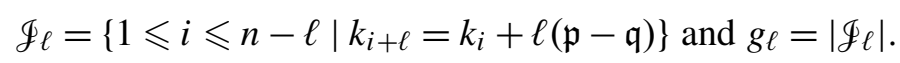

Corollary 2. For any integer $\ell \geqslant 0$, we have $g_{\ell} \leqslant \delta_{\ell} \leqslant n-a_{\ell}$.

Proof. The second inequality is a direct consequence of Corollary 1. Indeed, one has $k_{1}=0$ and $a_{\ell}=\operatorname{dim} \bar{M}_{a_{\ell}} \leqslant \operatorname{dim} \operatorname{ker} \delta^{\ell}$. For the first inequality, note that $\operatorname{rk} \delta_{\Lambda} \geqslant \sum_{i=0}^{s-1} \operatorname{rkgr}_{i+1}^{\mathscr{F}}\left(\delta_{\Lambda}\right)$. By Lemma 2, we get

$$
\delta_{1} \geqslant\left|\left\{1 \leqslant i \leqslant n-1 \mid k_{i+1}=k_{i}+\mathfrak{p}-\mathfrak{q}\right\}\right|=g_{1} .
$$

Since $\delta_{\Lambda}$ is strictly upper block-triangular, we have $\operatorname{rk} \delta_{\Lambda}^{\ell} \geqslant \sum_{i=0}^{s-\ell} \mathrm{rk} \mathrm{gr}_{i+\ell}^{\mathscr{F}}\left(\delta_{\Lambda}^{\ell}\right)$ for

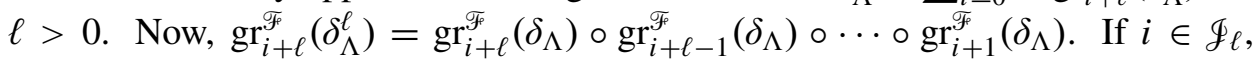
then $\operatorname{dim} \mathscr{F}_{i+1} / \mathscr{F}_{i}=\cdots=\operatorname{dim} \mathscr{F}_{i+\ell} / \mathscr{F}_{i+\ell-1}=1$. Since $\operatorname{gr}_{k}^{\mathscr{F}}\left(\delta_{\Lambda}\right)$ is nonzero for $i+1 \leqslant k \leqslant i+\ell$, the map $\operatorname{gr}_{i+\ell}^{\mathscr{T}}\left(\delta_{\Lambda}^{\ell}\right)$ is also nonzero, and $\operatorname{rk} \delta_{\Lambda}^{\ell} \geqslant g_{\ell}$ follows. 
1.2. The proof of Proposition 1. The proof relies on two combinatorial results.

Lemma 3. Let $T=\left(t_{1}, \ldots, t_{n}\right)$ be an increasing sequence of integers such that $t_{1}=0$ and $t_{i+1}-t_{i} \leqslant 1$. Let $J_{\ell}=\left\{1 \leqslant i \leqslant n-\ell \mid t_{i+\ell}=t_{i}+\ell\right\}$ and $c_{\ell}=\left|J_{\ell} \backslash J_{\ell+1}\right|$. Then we have

$$
\frac{1}{2} \sum_{j \geqslant 0} c_{j}\left(c_{j}-1\right) \leqslant \sum_{i \in J_{0} \backslash J_{1}}(n-i) \leqslant c_{0} \sum_{j \geqslant 0} c_{j}-\frac{1}{2} \sum_{j \geqslant 0} c_{j}\left(c_{j}-1\right) .
$$

Proof. Let $J_{0} \backslash J_{1}=\left\{h_{1}, \ldots, h_{c_{0}}\right\}$ with $h_{0}=0<h_{1}<\cdots<h_{c_{0}}=n$, and let $R=\sum_{i \in J_{0} \backslash J_{1}}(n-i)$. Clearly, $R=\sum_{i=1}^{c_{0}}\left(n-h_{i}\right)=\sum_{i=0}^{c_{0}} i\left(h_{i+1}-h_{i}\right)$. The interval $I=[0, n]$ is cut into $c_{0}$ segments of respective lengths $h_{i+1}-h_{i}$ for $0 \leqslant i \leqslant c_{0}-1$. By construction, there are $\left|\left\{1 \leqslant i \leqslant c_{0} \mid h_{i}-h_{i-1}=j\right\}\right|=c_{j-1}-c_{j}$ segments of length $j$. The quantity $R$ is the sum of these lengths weighted by their index of appearance along the interval $I$; therefore it is minimal (respectively maximal) when the segments are ordered by decreasing (respectively increasing) length. Thus, we get the bounds

$$
\sum_{j \geqslant 1} j \sum_{i=c_{j}}^{c_{j-1}-1} i \leqslant R \leqslant \sum_{j \geqslant 1} j \sum_{i=c_{0}-c_{j-1}}^{c_{0}-c_{j}-1} i
$$

A direct computation yields the claimed result.

For an integer sequence $\left(g_{i}\right)_{i \geqslant 0}$, let $\left(\Delta g_{i}\right)_{i \geqslant 0}$ be defined by $\Delta g_{i}=g_{i}-g_{i+1}$. Let us say that $\left(g_{i}\right)_{i \geqslant 0}$ is strongly decreasing if both $\left(g_{i}\right)_{i \geqslant 0}$ and $\left(\Delta g_{i}\right)_{i \geqslant 0}$ are nonincreasing. Let $\Delta g=\sum_{i \geqslant 0} \Delta g_{i}\left(\Delta g_{i}-1\right)$. Note that

$$
\Delta g=g_{0}^{2}-2 \sum_{j \geqslant 0} g_{j+1} \Delta g_{i}
$$

Lemma 4. Let $\left(g_{i}\right)_{i \geqslant 0}$ and $\left(\delta_{i}\right)_{i \geqslant 0}$ be two integer strongly decreasing sequences such that $g_{0}=\delta_{0}$ and $\delta_{i} \geqslant g_{i}$ for all $i \geqslant 0$. Then $\Delta g \geqslant \Delta \delta$.

Proof. By formula (4), one has $h=\frac{1}{2}(\Delta g-\Delta \delta)=\sum_{i \geqslant 1}\left(\delta_{i}-g_{i}\right)\left(\Delta \delta_{i-1}-\Delta g_{i}\right)$. Write $\Delta \delta_{i-1}-\Delta g_{i}=\left(\delta_{i-1}-g_{i-1}\right)-\left(\delta_{i}-g_{i}\right)+\Delta g_{i-1}-\Delta g_{i}$. Substituting in $h$, we straightforwardly get $h=\sum_{i \geqslant 1}\left(\delta_{i}-g_{i}\right)\left(\Delta g_{i-1}-\Delta \delta_{i}\right)$. Summing the two expressions for $h$, we get $2 h=\sum_{i \geqslant 1}\left(\delta_{i}-g_{i}\right)\left(\Delta g_{i-1}-\Delta g_{i}+\Delta \delta_{i-1}-\Delta \delta_{i}\right) \geqslant 0$; hence $\Delta g \geqslant \Delta \delta$.

Proof of Proposition 1. Let us consider the sequence $\mathscr{K}=\left(k_{1}, \ldots, k_{n}\right)$ of elementary divisors of $\Lambda_{\mathfrak{q}}$ with respect to $\Lambda$ and let $d=\mathfrak{p}-\mathfrak{q}$. Recall that

$$
\left[\Lambda: \Lambda_{\mathfrak{q}}\right]=\operatorname{dim}_{\mathbb{C}} \Lambda / \Lambda_{\mathfrak{q}}=k_{1}+\cdots+k_{n} ;
$$

see [Corel 2004, Lemma 2.3, page 1371]. Define $\ell_{0}$ as the largest $\ell$ such that $a_{\ell}<n$, so that $1 \leqslant a_{1} \leqslant \cdots \leqslant a_{\ell_{0}}<n=a_{\ell_{0}+1}$. By definition of $a_{i}$, we get 
$\sum_{i=a_{j}+1}^{a_{j+1}} k_{i} \geqslant j d\left(a_{j+1}-a_{j}\right)$. According to Corollary 2, we have

$$
\left[\Lambda: \Lambda_{\mathfrak{q}}\right]=\sum_{j=0}^{\ell_{0}} \sum_{i=a_{j}+1}^{a_{j+1}} k_{i} \geqslant \sum_{j=0}^{\ell_{0}} j\left(a_{j+1}-a_{j}\right) d=\sum_{j=1}^{\ell_{0}}\left(n-a_{j}\right) d \geqslant d \sum_{j=1}^{\ell_{0}} \delta_{j},
$$

which proves the left side of the proposition. For $1 \leqslant i \leqslant n-1$, let $d_{i}=k_{i+1}-k_{i}$. Since $d_{i} \leqslant d-1$ whenever $i \notin \mathscr{F}_{1}$, we get

$$
\sum_{i=1}^{n} k_{i}=\sum_{i=1}^{n-1}(n-i) d_{i} \leqslant \frac{1}{2} n(n-1) d-\sum_{i \notin \mathscr{F}_{1}}(n-i) .
$$

We wish to minimize $R=\sum_{i \in \mathscr{F}_{0} \backslash \mathscr{F}_{1}}(n-i)$. Construct the following sequence $T=\left(t_{1}, \ldots, t_{n}\right)$ defined by induction as

$$
\left\{\begin{aligned}
t_{1} & =0, \\
t_{i+1} & =t_{i}+\left[\left(k_{i+1}-k_{i}\right) / d\right], \quad \text { where }[x] \text { denotes the integer part of } x .
\end{aligned}\right.
$$

The sequence $T$ satisfies the assumptions of Lemma 3 with $c_{i}=\Delta g_{i}$; hence

$$
R \geqslant \frac{1}{2} \sum_{j \geqslant 0} \Delta g_{j}\left(\Delta g_{j}-1\right)
$$

Let $n_{i}$ be the number of Jordan blocks of size $i$ in the Jordan normal form of $\delta$. We have

$$
\delta_{i}=\sum_{j \geqslant i+1}(j-i) n_{j} \quad \text { and } \quad \Delta_{i}=\delta_{i}-\delta_{i+1}=\sum_{j \geqslant i+1} n_{j} \quad \text { for all } i \geqslant 0 .
$$

Accordingly, we have $\delta_{i} \geqslant \delta_{i+1}$ and $\Delta_{i} \geqslant \Delta_{i+1}$ for all $i \geqslant 0$. Let $\Gamma_{i}=g_{i}-g_{i+1}$. Both $g_{i} \geqslant g_{i+1}$ and $\Gamma_{i} \geqslant \Gamma_{i+1}$ hold, whereas Corollary 2 ensures that $g_{i} \leqslant \delta_{i}$. Both sequences are strongly decreasing, and $g_{0}=n=\delta_{0}$. Therefore, by Lemma 4 , we get $\Gamma=\sum_{j \geqslant 0} \Gamma_{j}\left(\Gamma_{j}-1\right) \geqslant \Delta=\sum_{j \geqslant 0} \Delta_{j}\left(\Delta_{j}-1\right)$. According to (5), we have $R \geqslant \Gamma$, so $R \geqslant \Delta$, thereby establishing the right side of the proposition.

1.3. The Levelt bundle. Let $(\mathscr{E}, \nabla)$ be a meromorphic connection, and $(V, \Lambda, \nabla)$ be the local model at $x \in X$. Let $\nabla=\nabla^{\text {reg }}+\omega$ be the formal Babbitt-Varadarajan decomposition of $\nabla$ into a regular formal connection $\nabla^{\text {reg }}$ and the $K$-linear map $\omega: V \rightarrow V \otimes_{K} \Omega$ that we introduced in [Corel 2004, Theorem 3]. By a slight abuse of terminology, we will act as if $\omega$ (which we call an irregular part of $\nabla$ at $x$ from now on) were an endomorphism of $V$ with coefficients in $\Omega$, and speak of its eigenspaces, eigenvalues, and so on. Although the Babbitt-Varadarajan decomposition depends on the choice of a local coordinate, we can retrieve from it the following invariants of $\nabla$ at $x$ :

- the ramification index $\rho_{x}(\nabla) \in \mathbb{N}$, equal to the splitting degree of $\omega$; 
- the Katz rank $\kappa_{x}(\nabla)=\max \left(-v_{x}\left(\omega_{1}\right)-1, \ldots,-v_{x}\left(\omega_{n}\right)-1\right) \in \mathbb{Q}$;

- the Malgrange irregularity $i_{x}(\nabla)=\sum_{i=1}^{n}\left(-v_{x}\left(\omega_{i}\right)-1\right) \in \mathbb{Q}$.

Here $v_{x}$ is the valuation defined by $\left(\mathrm{O}_{X}\right)_{x}$ (extended to formal ramified 1-forms in the natural way). We'll use the same notation for the local model at a given point.

Definition 1. Let $(\mathscr{E}, \nabla)$ be a meromorphic connection. Let $x \in X$, let $(V, \Lambda, \nabla)$ be the local model of $(\mathscr{E}, \nabla)$, and let $\nabla=\nabla^{\text {reg }}+\omega$ be a Babbitt-Varadarajan decomposition of $\nabla$ at $x$. The vector bundle $\mathscr{E}$ is log-split at $x$ if

(i) $\rho_{x}(\nabla)=1$, that is, $\nabla$ is unramified at $x$;

(ii) $\nabla^{\mathrm{reg}}(\Lambda) \subset \Lambda \otimes_{0} \Omega(1)$, that is, $\Lambda$ is logarithmic with respect to $\nabla^{\mathrm{reg}}$;

(iii) $\bigoplus_{i=1}^{s} \Lambda \cap V_{i}=\Lambda$, where $V_{1}, \ldots, V_{s}$ the eigenspaces of $\omega$.

We will next use a classical fact; see [Van der Put and Singer 2003, page 166].

Lemma 5. Let $\mathscr{E}$ be endowed with a meromorphic connection $\nabla$. For any family of lattices $M(x) \in \mathscr{E}_{X} \otimes_{\mathcal{O}_{X, x}} K\left(\mathscr{O}_{X, x}\right)$ for $x$ in a discrete set $\mathscr{Y}$, there exists a unique vector bundle $\mathscr{E}^{M}$ such that

$$
\left(\mathscr{E}^{M}\right)_{x}= \begin{cases}\mathscr{E}_{x} & \text { if } x \notin \mathscr{Y}, \\ M(x) & \text { if } x \in \mathscr{S}\end{cases}
$$

and a canonical meromorphic connection on $\mathscr{E}^{M}$ that coincides with $\nabla$ over $X \backslash \mathscr{Y}$. We still denote it by $\nabla$ although it may have additional singularities on $Y$.

Let $\Xi(\mathscr{E})$ denote the set of bundles obtained from $\mathscr{E}$ in this way. A natural extension of the local notion of index recalled in Section 1.1 to the set $\Xi(\mathscr{E})$ is

$$
[\mathscr{E}: \mathscr{F}]=\operatorname{deg} \mathscr{E}-\operatorname{deg} \mathscr{F}-n \sum_{x \in X} v_{\mathscr{E}_{x}}\left(\mathscr{F}_{x}\right) \quad \text { for } \mathscr{F} \in \Xi(\mathscr{E}) .
$$

Theorem 1. Let $\mathscr{E}$ be a holomorphic vector bundle equipped with a connection $\nabla$ that is unramified everywhere. There exists a unique maximal subsheaf $\mathscr{E}_{L}$ of locally free $\mathrm{O}_{X}$-modules of rank $n$ of $\mathscr{E}$ that is log-split everywhere. The sheaf $\mathscr{E}_{L}$ is called the Levelt bundle of the vector bundle $\mathscr{E}$.

Proof. At every singularity $x$, we can construct the formal Levelt lattice

$$
\hat{L}=\left(\mathscr{E}_{x} \otimes_{\mathscr{O}_{X}} \widehat{\mathrm{O}_{X, x}}\right)_{L}
$$

of the formalized stalk of $\mathscr{E}$ at $x$; see [Corel 2004, Proposition 4.1 and Definition 14, page 1384]. The Levelt bundle $\mathscr{E}_{L}$ is then simply constructed as the extension of $\mathscr{E}$ by the family of lattices $L_{x}=\hat{L} \cap\left(\mathscr{E}_{x} \otimes_{\mathcal{O}_{X}} K\left(\mathscr{O}_{X, x}\right)\right)$ for $x \in \mathscr{T}$.

Definition 2. Let $\tilde{X}$ be a compact Riemann surface. A nonconstant holomorphic map $\tilde{X} \stackrel{\pi}{\rightarrow} X$ is called a $\nabla$-admissible cover of $X$ if the pull-back connection $\pi^{*} \nabla$ on $\pi * \mathscr{E}$ over $\tilde{X}$ is unramified everywhere. 
Proposition 2. There exists a $\nabla$-admissible cover of $X$ for any connection $\nabla$.

Proof. Let $\mathscr{S}=\left\{x_{1}, \ldots, x_{p}\right\}$ be the singular set of $\nabla$ on $X$, and let $\rho_{i}=\rho_{x_{i}}(\nabla)$ be the ramification index at $x_{i}$ as in Definition 1 . Let $m=\operatorname{lcm}\left(\rho_{1}, \ldots, \rho_{p}\right)$, and let $g$ be a cycle of maximal length $m$ in the symmetric group $S_{m}$. If $p$ is even, let $g_{i}=g^{(-1)^{k-1}}$ for $1 \leqslant i \leqslant p$. If $p=2 q+1$ is odd, embed $S_{m}$ into $S_{2 m}$ as the subgroup fixing the last $m$ terms (in the standard action on $\{1, \ldots, 2 m\}$ ). Let $\tilde{g}$ be the cycle acting as $g$ on those last $m$ terms. Define now $g_{i}=g^{(-1)^{k-1}}$ for $1 \leqslant i \leqslant 2 q-1$, and put $g_{p-1}=\tilde{g}$ and $g_{p}=(g \tilde{g})^{-1}$. By construction, we have $g_{1} \cdots g_{p}=1$, and each permutation $g_{i}$ is an indecomposable cycle of length $m$, except at most one which appears as the product of two disjoint cycles of length $m$. By Riemann's existence theorem (see [Lando and Zvonkin 2004, Theorem 1.8.14 and Remark 1.8.15, pages 74-75]), there exist a compact Riemann surface $\tilde{X}$ and a holomorphic covering $\tilde{X} \stackrel{\pi}{\longrightarrow} X$, ramified over $\mathscr{S}=\left\{x_{1}, \ldots, x_{p}\right\}$, such that the order of ramification at all points of $\pi^{-1}(\mathscr{S})$ is equal to $m$, which is divisible by the corresponding $\rho_{i}$. Therefore, the covering map $\pi$ is $\nabla$-admissible. Note that the degree of $\pi$ is $m$ in the even case and $2 m$ in the odd one, and moreover that each fiber over $\mathscr{Y}$ is of cardinality 1 , except maybe at one point, where it is at most 2 .

At the cost of introducing an apparent ramification point $x_{0} \notin \mathscr{Y}$, one can impose that the ramification of $\pi$ be equal to the ramification of the connection, as follows. For $\rho, m \in \mathbb{N}$, let the regular $(\rho, m)$-permutation in $S_{\rho m}$ be defined as the product $g=c_{1} \cdots c_{m}$ of the $m$ disjoint cycles

$$
c_{k}=\left(\begin{array}{lll}
k \rho+1 & \cdots & (k+1) \rho \\
k \rho+2 & \cdots & k \rho+1
\end{array}\right)
$$

of length $\rho$. Let $N=\operatorname{lcm}\left(\rho_{1}, \ldots, \rho_{p}\right)$, and for all $1 \leqslant i \leqslant p$, let $m_{i}=N / \rho_{i}$. Define $g_{i} \in S_{N}$ as the regular $\left(\rho_{i}, m_{i}\right)$-permutation in $S_{N}$. The group $G=\left\langle g_{i}\right\rangle$ generated by these permutations acts transitively since $N$ is the $1 \mathrm{~cm}$ of the cycle lengths. For an arbitrary point $x_{0} \notin \mathscr{S}$, put $g_{0}=\left(g_{1} \cdots g_{p}\right)^{-1}$. Let $\tilde{X} \stackrel{\pi}{\longrightarrow} X$ be the ramified cover attached to the data $\left(x_{0}, \ldots, x_{p}, g_{0}, \ldots, g_{p}\right)$, as in the proof of Proposition 2 . In this case, the fiber $\pi^{-1}\left(x_{i}\right)$ for $i \geqslant 1$ consists of exactly $m_{i}$ points, each of ramification index $\rho_{i}$. For further reference, we call this special covering the minimal $\nabla$-admissible covering with apparent ramification at $x_{0}$.

Proposition 3. Let $(\mathscr{E}, \nabla)$ be an unramified connection on $X$, and let $\mathscr{E}_{L}$ be the Levelt bundle of $\mathscr{\mathscr { C }}$. For any covering map $\tilde{X} \stackrel{\pi}{\rightarrow} X$, we have $\left(\pi^{* \mathscr{E}}\right)_{L}=\pi^{*}\left(\mathscr{E}_{L}\right)$.

Proof. We only need to prove the local version of this fact. Let $\tilde{X}$ be a compact Riemann surface, and let $\tilde{X} \stackrel{\pi}{\longrightarrow} X$ be a nonconstant holomorphic map. For $\tilde{x} \in \tilde{X}$, the fraction field $H$ of the formal local ring $O_{H}=\widehat{\widehat{O}_{\tilde{X}}, \tilde{x}}$ is a finite extension of the fraction field $K$ of $\mathcal{O}=\widehat{O_{X, x}}$ of degree $m=e_{\tilde{x}}(\pi)$. The local model $(W, M, \Delta)$ of $\left(\pi^{* \mathscr{E}}, \pi^{*} \nabla\right)$ satisfies $W=V \otimes_{K} H$ and $M=\Lambda \otimes_{\mathcal{O}} \mathscr{O}_{H}$. In other terms, $(W, M, \Delta)$ 
is a degree $m$ finite extension of $(V, \Lambda, \nabla)$. Assume that $\nabla$ is unramified at $x$. The stalk $\Lambda_{L}=\left(\mathscr{E}_{L}\right)_{x}$ is the Levelt lattice of $\Lambda=\mathscr{E}_{x}$. We want to prove that the Levelt lattice $M_{L}=\left(\left(\pi^{* \mathscr{C}}\right)_{L}\right)_{\tilde{x}}$ of $M$ satisfies $M_{L}=\Lambda_{L} \otimes_{\mathcal{O}} \mathscr{O}_{H}$. We first assume that $\nabla$ is regular. The Levelt lattice $\Lambda_{L}$ is then simply the largest logarithmic sublattice of $\Lambda$. Suppose that $\left(\Lambda_{L}\right)_{H} \subsetneq M_{L}$. The lattice $L^{-}=M_{L} \cap V$ is the largest sublattice of $M_{L}$ (with the standard embedding $V \hookrightarrow W$ ) that is defined over $\mathbb{O}$, and it clearly satisfies $\nabla\left(L^{-}\right) \subset L^{-} \otimes_{\mathcal{O}} \Omega(1)$. By maximality of the Levelt lattice, we get $\Lambda_{L}=L^{-}$. By duality, the smallest 0 -rational lattice $L^{+}$containing $M_{L}$ also satisfies $\nabla\left(L^{+}\right) \subset L^{+} \otimes_{\mathcal{O}} \Omega(1)$. Therefore, we get the inclusions $L^{-}=\Lambda_{L} \subset L^{+} \subset \Lambda$. Since $\Lambda_{L}$ is maximal, we conclude that $L^{-}=L^{+}=\Lambda_{L}$; hence $M_{L}=\left(\Lambda_{L}\right)_{H}$ and we get the desired contradiction. If $\nabla$ is not regular, let $\nabla=\nabla^{r}+\omega$ be a Babbitt-Varadarajan decomposition of $\nabla$, and let $V_{H}=\bigoplus_{i=1}^{s} V_{i}$ be the direct sum of eigenspaces of $\omega$. According to [Corel 2004, proof of Proposition 4.1], the Levelt lattice $M_{L}$ satisfies $M_{L}=\bigoplus_{i=1}^{s} M_{L}\left(\nabla^{\text {reg }}\right) \cap V_{i}$, where $M_{L}\left(\nabla^{\mathrm{reg}}\right)$ is the Levelt lattice of $M$ with respect to the regular connection $\nabla^{\text {reg }}$. The first part of the proof then yields the claimed result.

Assume $\mathscr{E}$ is log-split with respect to the connection $\nabla$. Let $x \in X$ and let $\omega$ be an irregular part of $\nabla$ at $x$. The local model $(V, \Lambda, \nabla)$ of $\mathscr{E}$ at $x$ satisfies

$$
(\nabla-\omega)(\Lambda) \subset \Lambda \otimes_{0} \Omega(1) .
$$

Therefore $\langle\nabla-\omega, \theta\rangle$ induces a linear map $\Gamma$ on the $\mathbb{C}$-vector space $\Lambda / \mathfrak{m} \Lambda$ of dimension $n$, which is the residue of the regular connection $\nabla^{\text {reg }}$ with respect to $\Lambda$, and is independent of the irregular part $\omega$.

Definition 3. The exponents $\mu_{1}^{\mathscr{E}, x}, \ldots, \mu_{n}^{\mathscr{E}, x}$ at $x$ of the connection $(\mathscr{E}, \nabla)$ are

(1) the eigenvalues of the map $\Gamma$ defined just above if $\mathscr{E}$ is log-split and

(2) otherwise, the numbers

$$
\mu_{i}^{\mathscr{E}, x}=\frac{1}{e_{y}(\pi)} \mu_{i}^{\mathscr{E} L}(\pi), y \quad \text { for } 1 \leqslant i \leqslant n \text { for any } y \in \pi^{-1}(x),
$$

where $\tilde{X} \stackrel{\pi}{\longrightarrow} X$ is any $\nabla$-admissible ramified cover.

By definition, the Levelt bundle $\mathscr{E}_{L}(\pi)=\left(\pi^{* \mathscr{E}}\right)_{L}$ of $\pi^{* \mathscr{E}}$ is log-split with respect to $\pi^{*} \nabla$. According to Proposition 3, the previous definition makes sense.

\section{The companion bundles of a differential equation}

2.1. The Fuchs bundle. Let $\mathscr{S}$ be a discrete subset of $X$ and $U_{0}=X \backslash \mathscr{Y}$. For any $x_{i} \in \mathscr{Y}$ there exists an open neighborhood $U_{i}$ of $x_{i}$ such that $U_{i} \cap \mathscr{Y}=\left\{x_{i}\right\}$ and $U_{i} \cap U_{j}=\varnothing$ for nonzero $i \neq j$. The open covering $\mathfrak{U}=\left(U_{0}, \ldots, U_{p}\right)$ of $X$ will be called an adapted covering of $X$ (for $\mathscr{Y}$ ). 
Let $X$ be a compact Riemann surface, and let $K=\Gamma\left(X, \mu_{X}\right)$ be the function field of $X$. The set $\operatorname{Der}(K / \mathbb{C})=\Gamma\left(X, \Theta_{X}\right)$ of $\mathbb{C}$-derivations of $K$ and its dual $\Omega_{K / \mathbb{C}}^{1}=\Gamma\left(X, \mu_{X}^{1}\right)$ of differential forms are respectively the global sections of the sheaves of meromorphic sections of the holomorphic tangent and cotangent bundle of $X$. For any nontrivial derivation $\tau \in \operatorname{Der}(K / \mathbb{C})$, let $\omega_{\tau}$ be the unique differential form such that $\left\langle\omega_{\tau}, \tau\right\rangle=1$. Let $\mathfrak{D}_{X}=K[\tau]$. We consider a monic differential operator

$$
L=\tau^{n}+a_{1} \tau^{n-1}+\cdots+a_{n} \in \mathfrak{D}_{X}
$$

The singular set $\mathscr{Y}$ of (7) is the reunion of the supports of the divisor of $\tau$ and the polar divisors of $a_{1}, \ldots, a_{n}$.

We consider an adapted covering $\left(U_{0}, \ldots, U_{p}\right)$ of $X$ such that there is a local coordinate $t_{i}$ on $U_{i}$ for $i>0$. Locally around $x_{i}$, there exists a meromorphic function $f_{i} \in \Gamma\left(U_{i}, M_{X}\right)$ such that $f_{i} \tau=t_{i} \frac{d}{d t_{i}}$. We can assume, at the cost of shrinking $U_{i}$, that $f_{i}$ is holomorphic and does not vanish on $U_{i} \backslash\left\{x_{i}\right\}$. Put $\theta_{i}=t_{i} \frac{d}{d t_{i}}$. The operator $L_{i}=f_{i}^{n} L$ can be written on $U_{i}$ in the form

$$
L_{i}=\theta_{i}^{n}+b_{1}^{i} \theta_{i}^{n-1}+\cdots+b_{n}^{i} .
$$

Define differential systems defined over the subsets $U_{i}$ by

(0) $d f=\Omega_{0} f$ on $U_{0}$

(i) $d f=\Omega_{i} f$ on $U_{i}$ for $i>0 \quad$ with $\Omega_{i}=A_{L_{i}\left(\theta_{i}\right)} \otimes d t_{i} / t_{i}$,

where $A_{L}$ is the companion matrix of the operator $L$.

Lemma 6. The systems (S) can be glued together as a holomorphic vector bundle $\mathfrak{F}$ endowed with a meromorphic connection $\nabla$ on $X$, having exactly $\mathscr{Y}$ as singular set. We say that the pair $(\mathfrak{F}, \nabla)$ is the Fuchs bundle of the operator $L$.

Proof. By construction of the adapted covering $\left(U_{i}\right)$ of $X$, only the intersections $U_{0 i}=U_{0} \cap U_{i}$ are nonempty. Let $Q_{i}$ be the matrix whose $i$-th column represents the coefficients of $\theta_{i}$ in the basis $\left(1, \tau, \ldots, \tau^{n-1}\right)$. Also by construction, the matrix $P_{i}={ }^{t} Q_{i}^{-1}$ satisfies the relation $A_{L_{i}\left(\theta_{i}\right)}=\left(f_{i} / t_{i}\right) P_{i}^{-1} A_{L(\tau)} P_{i}-P_{i}^{-1} \theta_{i} P_{i}$. Restricting if necessary the open sets $U_{i}$, the matrix $P_{i}$ is holomorphically invertible in the set $U_{i} \backslash\left\{x_{i}\right\}=U_{0 i}$. Tensoring by $d t_{i} / t_{i}=\left(t_{i} / f_{i}\right) \omega_{\tau}$, we get

$$
\Omega_{i}=P_{i}^{-1} \Omega_{0} P_{i}-P_{i}^{-1} d P_{i} .
$$

The collection $\mathfrak{P}=\left(P_{i}\right)_{i=0}^{p}$ is a (̌̌ech) cocycle in $Z^{1}\left(\mathfrak{U}, \mathrm{GL}_{n}\left(\mathcal{O}_{X}\right)\right)$. Let $\mathbf{F}$ be a holomorphic vector bundle on $X$ having $\mathfrak{P}$ as cocycle, with $\mathfrak{F}$ its sheaf of sections. Then (8) defines a meromorphic connection $\nabla$ on $\mathfrak{F}$ with singularities only in $\mathscr{Y}$. 
2.2. The companion bundle. Let $(\mathfrak{F}, \nabla)$ be the Fuchs bundle of a differential operator $L \in \mathfrak{D}_{X}$ with singular set $\mathscr{Y}$. Let $\tilde{X} \stackrel{\pi}{\rightarrow} X$ be a $\nabla$-admissible ramified cover of $X$. We construct a holomorphic vector bundle $\mathfrak{C}(\pi)$ on $\tilde{X}$ as follows.

Let $\left(\pi^{*} \mathfrak{F}, \pi^{*} \nabla\right)$ be the pull-back of the Fuchs bundle. Let $\tilde{x} \in \pi^{-1}(\mathscr{Y})$ and consider the local model $(W, M, \Delta)$ of $\left(\pi^{*} \mathfrak{F}, \pi^{*} \nabla\right)$ at $\tilde{x}$. Let $K$ and $H$ be the field of fractions of $\mathcal{O}_{X, \pi(\tilde{x})}$ and $\mathrm{O}_{\tilde{X}, \tilde{x}}$, respectively. The degree of the extension $[\hat{H}: \hat{K}]=[H: K]$ is divisible by the ramification index of $\nabla$. Therefore $W$ admits a basis $(e)$ in which any irregular part is diagonal. Let $M$ be the $0_{\hat{H}}$-lattice spanned by $(e)$ in $W$. We define a local lattice $M(\tilde{x})$ at $\tilde{x}$ by putting $M(\tilde{x})=M \cap\left(\pi^{*} \mathfrak{F}\right) \tilde{x}$.

Definition 4. Let the companion bundle of $L$ with respect to the admissible ramified cover $\pi$ be the pair $\left(\mathfrak{C}(\pi), \nabla^{\pi}\right)$, where $\mathfrak{C}(\pi)$ is the continuation of the pull-back bundle $\left(\pi^{*} \mathfrak{F}, \pi^{*} \nabla\right)$ by the family of local lattices $(M(\tilde{x}))_{x \in \pi^{-1}(\mathscr{S})}$ and $\nabla^{\pi}$ is the extension of the pull-back connection $\pi^{*} \nabla$ to $\mathfrak{C}(\pi)$ according to Lemma 5 .

Note 1. When $L$ is fuchsian, the Fuchs bundle of $L$ coincides with the companion bundle $\mathfrak{C}\left(\mathrm{id}_{X}\right)$. However, the companion bundle of an irregular operator does not generally come from a bundle defined on $X-$ it is not even equal to the Levelt bundle $(\mathfrak{F})_{L}(\pi)$ of the Fuchs bundle.

The definition of Fuchs' exponents has been extended by D. Bertrand and G. Laumon [1985] in relation with arithmetic transcendence problems.

Theorem 2. Let $X=\mathbb{P}^{1}(\mathbb{C})$ and $L \in \mathbb{C}(z)\left[\frac{d}{d z}\right]$. The exponents $\mu_{j}^{\mathfrak{C}(\pi), \tilde{x}_{i}}\left(\nabla^{\pi}\right)$ of $\nabla^{\pi}$ with respect to the companion bundle $\mathfrak{C}(\pi)$ of an admissible ramified cover $\pi$ satisfy

$$
\mu_{j}^{\mathfrak{C}(\pi), \tilde{x}_{i}}\left(\nabla^{\pi}\right)=e_{\tilde{x}_{i}}(\pi) \lambda_{j}^{\pi\left(\tilde{x}_{i}\right)}(L),
$$

where the $\lambda_{j}^{\pi\left(\tilde{x}_{i}\right)}(L)$ are the Bertrand-Laumon exponents of $L$.

Proof. If the operator $L$ is fuchsian, the Fuchs bundle $\mathfrak{F}$ is logarithmic and the exponents $\mu_{j}^{\mathfrak{F}, x_{i}}(\nabla)$ of $\nabla$ with respect to $\mathfrak{F}$ are equal to the eigenvalues of the residue of $\Omega_{i}$. By construction, the characteristic polynomial of $\operatorname{Res}_{x_{i}} \Omega_{i}$ is indeed equal to the indicial polynomial $P_{L}^{x_{i}}$. If $L$ is not fuchsian, the bundle $\mathfrak{C}(\pi)$ is nevertheless log-split. At any $\tilde{x} \in \mathscr{S}\left(\nabla^{\pi}\right)$, consider the formal local model $(V, \Lambda, \nabla)$ of $\left(\mathfrak{C}(\pi), \nabla^{\pi}\right)$. There is an isomorphism of $\mathfrak{D}_{\hat{H}}$-modules

$$
\mathfrak{D}_{\hat{H}} / \mathfrak{D}_{\hat{H}} L \simeq \bigoplus_{i=1}^{t} \mathfrak{D}_{\hat{H}} / \mathfrak{D}_{\hat{H}} L_{i}\left(\theta_{\zeta}-\eta_{i}\right),
$$

where $L_{i}$ are formal fuchsian operators and $\eta_{i} \in(1 / \zeta) \mathbb{C}[(1 / \zeta)]$, where $\zeta$ is a uniformizing parameter of $H$, and $\theta_{\zeta}=\zeta \frac{d}{d \zeta}$; see [Robba 1980, théorème 3.2]. There exists an $H$-basis $(e)$ of $V$ and a local parameter $\zeta$ of $\tilde{X}$ such that the matrix 
of the irregular part $\omega$ of $\nabla$ attached to $\zeta$ at $\tilde{x}$ is the diagonal matrix

$$
\operatorname{Mat}\left(\omega_{H},(e)\right)=\operatorname{diag}\left(\omega_{1} I_{n_{1}}, \ldots, \omega_{s} I_{n_{t}}\right), \quad \text { where } \omega_{i}=\eta_{i} \otimes(d z / z) .
$$

As a consequence of [Corel 2004, proof of Theorem 3, page 1383], the map $\omega_{H}$ is in fact defined as the tensor extension of a map $\omega$ defined over $K$. Explicitly, $\operatorname{Mat}(\nabla-\omega,(e))=\operatorname{diag}\left(B_{L_{1}} \otimes(d \zeta / \zeta), \ldots, B_{L_{t}} \otimes(d \zeta / \zeta)\right)$. By definition, the exponents $\mu_{j}^{\mathfrak{C}(\pi), \tilde{x}_{i}}\left(\nabla^{\pi}\right)$ are the eigenvalues of $\operatorname{Res}_{\zeta=0} \operatorname{Mat}(\nabla-\omega,(e))$, and therefore the roots of the characteristic polynomials of

$$
\operatorname{Res}_{\zeta=0} B_{L_{i}}=e_{\tilde{x}_{i}}(\pi) \operatorname{Res}_{z=0} B_{L_{i}} .
$$

But this matches exactly the definition of the exponents of the operator $L$; see [Bertrand and Beukers 1985].

Corollary 3. The definition of the Bertrand-Laumon exponents of a differential operator $L \in \mathfrak{D}_{X}$ holds on any Riemann surface $X$, and so does Theorem 2 if $X$ is compact.

Indeed, the companion bundle and its exponents are independent of the local parameter. This answers a question raised originally by G. Laumon.

\section{Fuchs relations}

Theorem 3 (Fuchs relation). Let $\mathscr{E}$ be a holomorphic vector bundle equipped with a meromorphic connection $\nabla$. The sum of the exponents $\mu_{i}^{\mathscr{E}, x}$ of $\nabla$ with respect to $\mathscr{E}$ at all points $x \in X$ satisfies the relations

$$
\sum_{x \in X} \sum_{i=1}^{n} \mu_{i}^{\mathscr{E}, x}=\frac{\operatorname{deg} \mathscr{E}_{L}(\pi)}{\operatorname{deg} \pi} \leqslant \operatorname{deg} \mathscr{E},
$$

where $\mathscr{E}_{L}(\pi)$ is the Levelt bundle of $\mathscr{E}$ in any admissible ramified cover $\tilde{X} \stackrel{\pi}{\rightarrow} X$. Moreover, equality is achieved if and only if $\mathscr{E}$ is log-split.

Proof. Assume that $\mathscr{E}$ is log-split with respect to $\nabla$. Let $\bigwedge^{n} \nabla$ be the connection induced by $\nabla$ on the determinant line bundle $\bigwedge^{n} \mathscr{E}$. A line bundle is always log-split with respect to a connection on it, so Definition 3 gives $\sum_{i=1}^{n} \mu_{i}^{\mathscr{\ell}, x}=\operatorname{Res}_{x} \Lambda^{n} \nabla$. By Bott's [1967] residue formula for line bundles, we get $\sum_{x \in X} \operatorname{Res}_{x} \Lambda^{n} \nabla=$ $\operatorname{deg} \bigwedge^{n} \mathscr{E}=\operatorname{deg} \mathscr{E}$ and hence the result. In the general case, let $\tilde{X} \stackrel{\pi}{\longrightarrow} X$ be a $\nabla$-admissible cover. By definition, $\mu_{i}^{\mathscr{E}, x}=\mu_{i}^{\mathscr{E} L}(\pi), \tilde{x} / e_{\tilde{x}}(\pi)$ for any $\tilde{x} \in \pi^{-1}(x)$. Therefore, we get

$$
\sum_{\tilde{x} \in \pi^{-1}(x)} \sum_{i=1}^{n} \mu_{i}^{\mathscr{E}_{L}(\pi), \tilde{x}}=\sum_{\tilde{x} \in \pi^{-1}(x)} e_{\tilde{x}}(\pi) \sum_{i=1}^{n} \mu_{i}^{\mathscr{E}, x}=\operatorname{deg} \pi \sum_{i=1}^{n} \mu_{i}^{\mathscr{E}, x} .
$$


Since $\mathscr{E}_{L}(\pi)$ is $\log$-split, we get $\sum_{\tilde{x} \in \tilde{X}} \sum_{i=1}^{n} \mu_{i}^{\mathscr{E}_{L}(\pi), \tilde{x}}=\operatorname{deg} \mathscr{E}_{L}(\pi)$.

Now assume that $\nabla$ is unramified. The exact sequence $0 \rightarrow \mathscr{E}_{L} \rightarrow \mathscr{E} \rightarrow \mathfrak{P} \rightarrow 0$ yields the following relation for the dimensions of the cohomology groups:

$$
h^{0}\left(X, \mathscr{E}_{L}\right)-h^{0}(X, \mathscr{E})+h^{0}(X, \mathfrak{P})-h^{1}\left(X, \mathscr{E}_{L}\right)+h^{1}(X, \mathscr{E})-h^{1}(X, \mathfrak{P})=0
$$

By the Riemann-Roch theorem for a vector bundle $\mathscr{F}$ [Gunning 1967, page 64], we have $h^{0}(X, \mathscr{F})-h^{1}(X, \mathscr{F})=\operatorname{deg} \mathscr{F}+n(1-g)$, where $g$ is the genus of $X$. The sheaf $\mathfrak{P}$ is a skyscraper sheaf with support on $\mathscr{Y}$; thus $H^{1}(X, \mathfrak{P})=0$. From (9), we get

$$
\operatorname{deg} \mathscr{E}-\operatorname{deg} \mathscr{E}_{L}=h^{0}(X, \mathfrak{P})=\sum_{x \in \mathscr{S}} \operatorname{dim}_{\mathbb{C}}\left(\mathscr{E} / \mathscr{E}_{L}\right)_{x}=\sum_{x \in \mathscr{S}}\left[\mathscr{E}_{x}:\left(\mathscr{E}_{x}\right)_{L}\right] \geqslant 0
$$

and hence the inequality. So, $\operatorname{deg} \mathscr{E}=\operatorname{deg} \mathscr{E}_{L}$ if and only if $\mathscr{E}_{x}=\left(\mathscr{E}_{x}\right)_{L}$ for all $x \in \mathscr{Y}$, that is, $\mathscr{E}=\mathscr{E}_{L}$. The ramified case follows since $\operatorname{deg} \pi \operatorname{deg} \mathscr{E}=\operatorname{deg} \pi * \mathscr{E}$.

This result shows that log-split is a sensible extension to the irregular case of the classical notion of logarithmic vector bundle for a regular connection. The maximality property stated in Theorem 1 together with relation (10) yield the following consequence, and show that the exponents also measure how far the vector bundle $\mathscr{E}$ is from being log-split.

Corollary 4. For any $\nabla$-admissible covering $\pi$ of $X$, and any log-split bundle $\mathscr{F} \in \Xi\left(\pi^{* \mathscr{E}}\right)$, one has

$$
0 \leqslant d_{\nabla}(\mathscr{E})=\operatorname{deg} \mathscr{E}-\sum_{x \in X} \sum_{i=1}^{n} \mu_{i}^{\mathscr{E}, x} \leqslant \frac{1}{\operatorname{deg} \pi}\left[\pi^{* \mathscr{E}: \mathscr{F}}\right],
$$

and the minimum is attained only for the Levelt bundle $\mathscr{E}_{L}(\pi)$ for a given covering $\pi$. The nonnegative rational number $d_{\nabla}(\mathscr{E})$ will be called the $\nabla$-defect of the bundle $\mathscr{E}$.

Corollary 5. Let $\mathfrak{C}(\pi)$ be the companion bundle of a differential operator $L \in \mathfrak{D}_{X}$ with respect to a ramified cover $\pi$ of $X$. The degree $\operatorname{deg} \mathfrak{C}(\pi)$ satisfies the relation

$$
\operatorname{deg} \mathfrak{C}(\pi)=\left(\frac{1}{2} n(n-1)(p+2 g-2)+\frac{1}{2} i(\text { End } L)\right) \operatorname{deg} \pi,
$$

where $g$ is the genus of $X$ and $i($ End $L)=\sum_{x \in X} i_{x}($ End $L)$ is the total irregularity of the operator End $L$ induced by $L$ on End $\mathfrak{D}_{X}$. Therefore, a companion bundle is never trivial except if $L$ is regular with $p=2$ singularities over $X=\mathbb{P}^{1}(\mathbb{C})$.

Proof. We have $\sum_{\tilde{x} \in \tilde{X}} \sum_{j=1}^{n} \mu_{j}^{\mathfrak{C}(\pi), \tilde{x}}(\nabla)=\operatorname{deg} \mathfrak{C}(\pi)$ since the bundle $\mathfrak{C}(\pi)$ is log-split with respect to $\nabla$. On the other hand, the generalized Fuchs relation on a 
Riemann surface (see [Saito 1958] or [Chudnovsky and Chudnovsky 1984]) asserts that

$$
\sum_{x \in \mathscr{S}(L)}\left(\sum_{j=1}^{n} \lambda_{j}^{x}(L)-\frac{1}{2} i_{x}(\text { End } L)\right)=(p+2 g-2) \frac{1}{2} n(n-1) .
$$

A vector bundle $\mathscr{F}$ is trivial only if it has degree 0 . The only case when this can happen for $\mathfrak{C}(\pi)$ is for a fuchsian operator on $\mathbb{P}^{1}(\mathbb{C})$ with $p=2$, and it does indeed since the Fuchs bundle over $\mathbb{P}^{1}(\mathbb{C})$ is isomorphic to $\bigoplus_{i=0}^{n-1} \mathbb{O}(-i(p-2))$; see [Van der Put and Singer 2003, Proposition 6.14].

Note 2. In the case of $\mathbb{P}^{1}(\mathbb{C})$, Bolibrukh [1990a] constructs a Fuchsian system from a fuchsian operator $L$, whose exponents $\mu_{i}^{s}$ coincide with the exponents $\lambda_{i}^{s}$ of $L$, except at one point $x$, where $\mu_{i}^{x}=\lambda_{i}^{x}-i(p-2)$ for $1 \leqslant i \leqslant n$. This system can be understood as a meromorphic trivialization of the Fuchs bundle $\mathfrak{F}$ of $L$, which is holomorphic outside $x$ and moreover logarithmic at $x$, the correction to the exponents at $x$ corresponding precisely to the type of the bundle.

\subsection{The local index of the Levelt bundle.}

Definition 5. Let $f \in \operatorname{End}\left(\mathbb{C}^{n}\right)$, let $\left\{\lambda_{1}, \ldots, \lambda_{t}\right\}$ be the distinct eigenvalues of $f$, and let $E_{i}=\operatorname{ker}\left(f-\lambda_{i} \mathrm{id}_{\mathbb{C}^{n}}\right)^{n}$ be the characteristic space corresponding to $\lambda_{i}$. Put finally $\rho_{i}^{j}=\operatorname{rk}\left(\left.f\right|_{E_{i}}-\lambda_{i} \mathrm{id}_{E_{i}}\right)^{j}$. We define the Jordan invariant $J(f)$ and the kernel invariant $K(f)$ of $f$ as the quantities

$$
J(f)=\sum_{d \geqslant 1} \frac{1}{2} d(d-1) n_{d}=\sum_{i=1}^{t} \sum_{j \geqslant 1} \rho_{i}^{j} \quad \text { and } \quad K(f)=\frac{1}{2} \sum_{i=1}^{t} \sum_{j \geqslant 0} \Delta_{i}^{j}\left(\Delta_{i}^{j}-1\right),
$$

where $n_{d}$ is the number of Jordan blocks of dimension $d$ in the Jordan normal form of $f$, and $\Delta_{i}^{j}=\rho_{i}^{j}-\rho_{i}^{j+1}$.

Let $(V, \Lambda, \nabla)$ be the local model of $(\mathscr{E}, \nabla)$ at a point $x \in \mathscr{Y}(\nabla)$, with corresponding field $K$, ring $\mathcal{O}$ and maximal ideal $\mathfrak{m}$. Let $\Lambda_{L}$ be the Levelt lattice of $\Lambda$. Take a representative $\delta=\delta_{\Lambda}$ of $(P M \nabla)_{x}$, and let $E_{1}, \ldots, E_{t} \subset \Lambda / \mathfrak{m} \Lambda$ be the characteristic spaces corresponding to the different eigenvalues of $\delta$. By Sibuya's lemma (see [Babbitt and Varadarajan 1983, Lemma 1, page 42]), the space $V$ is a direct sum of uniquely defined $\nabla$-stable sub- $K$-vector spaces $\left\{W_{1}, \ldots, W_{t}\right\}$ of $V$, such that $\left(W_{i} \cap \Lambda\right) / \mathfrak{m} \Lambda=E_{i}$. Let $\nabla_{i}=\left.\nabla\right|_{W_{i}}$ and $\delta_{i}=\left.\delta\right|_{W_{i}}$. The formal connections $\left(W_{i}, \nabla_{i}\right)_{1 \leqslant i \leqslant t}$ form the Sibuya splitting of $(\mathscr{E}, \nabla)$ at $x$. Note that we have $\bigoplus_{i=1}^{t} W_{i} \cap \Lambda=\Lambda$ and $\Lambda_{L}=\bigoplus_{i=1}^{t}\left(W_{i} \cap \Lambda\right)_{L}$ [Corel 2004, page 1389].

Proposition 4. If the connection $\nabla$ is unramified at $x$, the index $\left[\Lambda: \Lambda_{L}\right]$ of the Levelt lattice $\Lambda_{L}$ of $\Lambda$ satisfies

$$
(\mathfrak{p}-\kappa) J(\delta) \leqslant \sum_{i=1}^{t}\left(\mathfrak{p}-\kappa_{i}\right) J\left(\delta_{i}\right) \leqslant\left[\Lambda: \Lambda_{L}\right] \leqslant \frac{1}{2} n(n-1) \mathfrak{p}-\frac{1}{2} i_{x}(\mathscr{E} \cap \ln \nabla)-K(\delta),
$$


where $\mathfrak{p}=\mathfrak{p}_{x}(\mathscr{E}$ nd $\nabla), \kappa=\kappa_{x}(\mathscr{E}$ nd $\nabla)$ and $\kappa_{i}=\kappa\left(\right.$ End $\left.\nabla_{i}\right)$.

Proof. Since $\nabla$ is unramified at $x$, the Katz rank $\kappa$ is an integer and we have

$$
\left[\Lambda: \Lambda_{L}\right]=\left[\Lambda: \Lambda_{\kappa}\right]+\left[\Lambda_{\kappa}: \Lambda_{L}\right]
$$

On the other hand, by Sibuya's lemma, we have $\left[\Lambda: \Lambda_{L}\right]=\sum_{i=1}^{t}\left[\Lambda_{i}:\left(W_{i} \cap \Lambda\right)_{L}\right]$. We can therefore restrict to the case where the map $\delta$ has only one eigenvalue $\lambda$. Let $\omega_{i}=\varphi_{i} d z / z$ be the eigenvalues of the irregular part of $\nabla$ at $x$ attached to a local coordinate $z$. For $Q=a_{k} z^{k}+\cdots+a_{\ell} z^{\ell} \in \mathbb{C}\left[z, z^{-1}\right]$, put $v(Q)=k$ and $\operatorname{deg} Q=\ell$. If $\lambda \neq 0$, then there exists a unique $P \in(1 / z) \mathbb{C}[1 / z]$ of largest degree such that $v\left(\varphi_{i}-P\right)>v\left(\varphi_{i}\right)$ for all $i$. If $\lambda=0$, it can also happen that the polynomial $P$ is defined. Otherwise, put $P=0$. The map $\nabla^{\prime}=\nabla-P d z / z$ is also a connection on $V$. According to [Corel 2004, Lemma 4.2], the Levelt lattices $\Lambda_{L}(\nabla)$ and $\Lambda_{L}\left(\nabla^{\prime}\right)$ coincide. Let $\mathfrak{p}^{\prime}$ and $\kappa^{\prime}$ respectively be the Poincaré and Katz ranks of $\nabla^{\prime}$. From relation (11) we get

$$
\left[\Lambda: \Lambda_{L}\right]=\left[\Lambda: \Lambda_{L}\left(\nabla^{\prime}\right)\right]=\left[\Lambda: \Lambda_{\kappa^{\prime}}\left(\nabla^{\prime}\right)\right]+\left[\Lambda_{\kappa^{\prime}}\left(\nabla^{\prime}\right): \Lambda_{L}\left(\nabla^{\prime}\right)\right] .
$$

According to [Corel 2004, proof of Proposition 5.1, page 1390], we have

$$
0 \leqslant\left[\Lambda_{\kappa^{\prime}}\left(\nabla^{\prime}\right): \Lambda_{L}\left(\nabla^{\prime}\right)\right] \leqslant \frac{1}{2} n(n-1) \kappa^{\prime}-\frac{1}{2} i\left(\text { End } \nabla^{\prime}\right),
$$

while Proposition 1 yields

$$
\left(\mathfrak{p}^{\prime}-\kappa^{\prime}\right) \sum_{j \geqslant 1} \rho^{j} \leqslant\left[\Lambda: \Lambda_{\kappa^{\prime}}\left(\nabla^{\prime}\right)\right] \leqslant \frac{1}{2} n(n-1)\left(\mathfrak{p}^{\prime}-\kappa^{\prime}\right)-\frac{1}{2} \sum_{j \geqslant 0} \Delta^{j}\left(\Delta^{j}-1\right),
$$

where $\rho^{j}=\operatorname{rk}\left(\delta_{\Lambda}-\lambda \operatorname{id}_{V}\right)^{j}$ and $\Delta^{j}=\rho^{j}-\rho^{j+1}$. Clearly $i\left(\right.$ End $\left.\nabla^{\prime}\right)=i($ End $\nabla)$ and $\mathfrak{p}^{\prime}$ and $\kappa^{\prime}$ respectively are equal to the Poincaré and Katz ranks of End $\nabla$. Adding these two inequalities, we get

$$
\left(\mathfrak{p}^{\prime}-\kappa^{\prime}\right) \sum_{j \geqslant 1} \rho^{j} \leqslant\left[\Lambda: \Lambda_{L}\right] \leqslant \frac{1}{2} n(n-1) \mathfrak{p}^{\prime}-\frac{1}{2} i(\text { End } \nabla)-\frac{1}{2} \sum_{j \geqslant 0} \Delta^{j}\left(\Delta^{j}-1\right) .
$$

for the case where the map $\delta_{\Lambda}$ has only one eigenvalue. If we have several eigenvalues $\lambda_{1}, \ldots, \lambda_{t}$, then $\mathfrak{p}^{\prime}=\mathfrak{p}$. Relation (11) implies

$$
\begin{aligned}
\sum_{i=1}^{t}\left(\mathfrak{p}_{i}-\kappa_{i}\right) \sum_{j \geqslant 1} \rho_{i}^{j} & \leqslant\left[\Lambda: \Lambda_{L}\right] \\
& \leqslant \sum_{i=1}^{t} \frac{1}{2} n_{i}\left(n_{i}-1\right) \mathfrak{p}_{i}-\sum_{i=1}^{t} \frac{1}{2} i\left(\text { End } \nabla_{i}\right)-\frac{1}{2} \sum_{i=1}^{t} \sum_{j \geqslant 0} \Delta_{i}^{j}\left(\Delta_{i}^{j}-1\right),
\end{aligned}
$$

where $n_{i}=\operatorname{dim}_{\mathbb{C}} E_{i}$ and $\mathfrak{p}_{i}$ is the Poincaré rank of the subconnection End $\nabla_{i}$ over the lattice $\Lambda \cap W_{i}$. However, we have $\mathfrak{p}_{i} \leqslant \mathfrak{p}$ and $\mathfrak{p}_{i}<\mathfrak{p}$ if and only if $J\left(\delta_{i}\right)=0$; 
therefore we can replace everywhere $\mathfrak{p}_{i}$ with $\mathfrak{p}$. By definition,

$$
K(\delta)=K\left(\delta_{\Lambda}\right)=\frac{1}{2} \sum_{i=1}^{t} \sum_{j \geqslant 0} \Delta_{i}^{j}\left(\Delta_{i}^{j}-1\right) \quad \text { and } \quad J\left(\delta_{i}\right)=\sum_{j \geqslant 1} \rho_{i}^{j} .
$$

The eigenvalues of the irregular part (determinant factors) of End $\nabla_{i}$ are among those of End $\nabla$; therefore we have $\kappa_{i} \leqslant \kappa$ and hence

$$
\sum_{i=1}^{t}\left(\mathfrak{p}-\kappa_{i}\right) J\left(\delta_{i}\right) \geqslant(\mathfrak{p}-\kappa) \sum_{i=1}^{t} J\left(\delta_{i}\right)=(\mathfrak{p}-\kappa) J(\delta),
$$

since $\delta=\bigoplus_{i=1}^{t} \delta_{i}$ and distinct $\delta_{i}$ have no common eigenvalue. Finally, by the argument used in [Corel 2004, top of page 1391], we get

$$
\sum_{i=1}^{t} \frac{1}{2} i\left(\text { End } \nabla_{i}\right)+\sum_{i<j} n_{i} n_{j} \mathfrak{p}=\frac{1}{2} i(\text { End } \nabla)=\frac{1}{2} i_{x}(\mathscr{E} \text { [nd } \nabla)
$$

R. R. Gontsov [2004] gives an analogous relation, with the weaker bounds rk $\delta$ instead of $J(\delta)$ and $\sum_{i=1}^{t} \Delta_{0}^{i}\left(\Delta_{0}^{i}-1\right)$ instead of $K(\delta)$.

\subsection{Fuchs inequalities for a holomorphic vector bundle.}

Definition 6. With the previous notation, we define

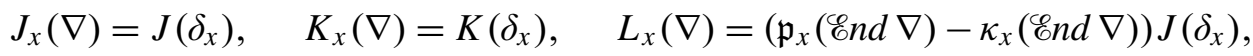

where $\delta_{x}$ is a representative of $(P M \nabla)_{x}$ if $\mathfrak{p}_{x}(\nabla)>0$, and $\delta_{x}=0$ otherwise.

Theorem 4 (Fuchs inequalities). Let $\nabla$ be a meromorphic connection over a vector bundle $\mathscr{E}$ of rank $n$. The sum of exponents satisfies the inequalities $\operatorname{deg} \mathscr{E}-\frac{1}{2} n(n-1) h(\mathscr{E} n d \mathscr{E})+\frac{1}{2} i(\mathscr{E} n d \nabla)+K(\mathscr{E}) \leqslant \sum_{x \in X} \sum_{i=1}^{n} \mu_{i}^{\mathscr{E}, x} \leqslant \operatorname{deg} \mathscr{E}-L(\mathscr{E})$, where

$$
L(\mathscr{E})=\sum_{x \in X} L_{x}(\nabla), \quad i(\mathscr{E} \cap n d \nabla)=\sum_{x \in X} i_{x}(\mathscr{E} \cap n d \nabla), \quad K(\mathscr{E})=\sum_{x \in X} \frac{1}{\rho_{x}(\nabla)} K_{x}(\nabla) .
$$

Proof. Assume $\nabla$ is unramified. Proposition 4 and relation (10) imply

$$
L(\mathscr{E}) \leqslant \operatorname{deg} \mathscr{\mathscr { E }}-\operatorname{deg} \mathscr{E}_{L} \leqslant \frac{1}{2} n(n-1) h(\mathscr{E} n d \mathscr{E})-\frac{1}{2} i(\mathscr{E} n d \nabla)-K(\mathscr{E})
$$

when $\nabla$ has no ramification, whence the result by Theorem 3. Let now $\tilde{X} \stackrel{\pi}{\rightarrow} X$ be an admissible ramified cover. The connection $\pi^{*} \nabla$ is unramified; hence we have

$$
\begin{aligned}
L\left(\pi^{* \mathscr{E}}\right) & \leqslant \operatorname{deg} \pi^{* \mathscr{E}}-\operatorname{deg} \mathscr{E}_{L}(\pi) \\
& \leqslant \frac{1}{2} n(n-1) h\left(\mathscr{E} n d \pi^{* \mathscr{E}}\right)-\frac{1}{2} i\left(\mathscr{E} n d \pi^{*} \nabla\right)-K\left(\pi^{* \mathscr{E}}\right) .
\end{aligned}
$$


Let $\tilde{x} \in \tilde{X}$ and put for simplicity $e=e_{\tilde{x}}(\pi)$ and $x=\pi(\tilde{x})$. If the map $(P M \nabla)_{x}$ has matrix $M$ for a given local basis of sections of $\mathscr{E}$ and a local coordinate at $x$, then there exist a local basis of sections of $\pi^{* \mathscr{E}}$ and a local coordinate at $\tilde{x}$ such that $\left(P M \pi^{*} \nabla\right)_{\tilde{x}}$ has matrix $e M$. Therefore $K_{\tilde{x}}\left(\pi^{* \mathscr{E}}\right)=K_{x}(\mathscr{E})$, and $K\left(\pi^{* \mathscr{E}}\right)=\sum_{x \in X}\left|\pi^{-1}(x)\right| K_{x}(\mathscr{E})$. If $\pi$ is the minimal covering with apparent ramification at $x_{0} \notin \mathscr{Y}$, we get $\left|\pi^{-1}(x)\right|=\rho_{x}(\nabla) \operatorname{deg} \pi$. Since $\mathscr{E}$ nd $\pi^{*} \nabla=\pi^{*} \mathscr{E} n d \nabla$ and $\mathfrak{p}_{\tilde{x}}\left(\pi^{*} \nabla\right)=e \mathfrak{p}_{x}(\nabla)$, we get $\kappa_{\tilde{x}}\left(\mathscr{E} \cap n d \pi^{*} \nabla\right)=e \kappa_{x}(\mathscr{E} n d \nabla)$ and $i_{\tilde{x}}\left(\mathscr{E} \cap n d \pi^{*} \nabla\right)=$ $e i_{x}(\mathscr{E}$ nd $\nabla)$. In particular, $J_{\tilde{x}}\left(\pi^{*} \nabla\right)=e J_{x}(\nabla)$. Therefore we get

$$
J\left(\pi^{* \mathscr{E}}\right)=\sum_{\tilde{x} \in \tilde{X}} e_{\tilde{x}}(\pi) J_{\pi(\tilde{x})}(\nabla)=\operatorname{deg} \pi \sum_{x \in X} J_{x}(\nabla)=\operatorname{deg} \pi J(\nabla) .
$$

The same holds for any subconnection; therefore $L\left(\pi^{* \mathscr{E}}\right)=(\operatorname{deg} \pi) L(\mathscr{E})$. In the same way, $h\left(\mathscr{E} n d \pi^{* \mathscr{E}}\right)=(\operatorname{deg} \pi) h(\mathscr{E} n d \mathscr{E})$ and $i\left(\mathscr{E} n d \pi^{*} \nabla\right)=(\operatorname{deg} \pi) i(\mathscr{E} n d \nabla)$. Since $\operatorname{deg} \pi * \mathscr{E}=\operatorname{deg} \pi \operatorname{deg} \mathscr{E}$, dividing out (12) by $\operatorname{deg} \pi$ gives the claim.

Corollary 6. The $\nabla$-defect of $\mathscr{E}$ satisfies

$$
L(\mathscr{E}) \leqslant d_{\nabla}(\mathscr{E}) \leqslant \frac{1}{2} n(n-1) h\left(\mathscr{E} n d^{\mathscr{E}}\right)-\frac{1}{2} i(\mathscr{E} n d \nabla)-K(\mathscr{E}) .
$$

Note 3. We can refine all these estimates by replacing the quantity $L(\mathscr{E})$ with $\ell(\mathscr{E})=\sum_{x \in X} \sum_{i=1}^{t}\left(\mathfrak{p}_{x}\left(\right.\right.$ End $\left.\nabla_{i}\right)-\kappa_{x}\left(\right.$ End $\left.\left.\nabla_{i}\right)\right) J_{x}\left(\nabla_{i}\right)$, where $\left(\nabla_{1}, \ldots, \nabla_{t}\right)$ are the local formal subconnections given by the Sibuya splitting of $\mathscr{E}$ at $x$.

\section{References}

[Babbitt and Varadarajan 1983] D. G. Babbitt and V. S. Varadarajan, "Formal reduction theory of meromorphic differential equations: A group theoretic view", Pacific J. Math. 109:1 (1983), 1-80. MR 86b:34010 Zbl 0533.34010

[Bertrand and Beukers 1985] D. Bertrand and F. Beukers, "Équations différentielles linéaires et majorations de multiplicités”, Ann. Sci. École Norm. Sup. (4) 18:1 (1985), 181-192. MR 87d:11052 Zbl 0578.12016

[Bertrand and Laumon 1985] D. Bertrand and G. Laumon, "Appendice à Exposants, vecteurs cycliques et majorations de multiplicités", preprint from C.R. conf. franco-japonaise (Strasbourg), IRMA, 1985.

[Bolibruch et al. 2006] A. A. Bolibruch, S. Malek, and C. Mitschi, "On the generalized RiemannHilbert problem with irregular singularities”, Expo. Math. 24:3 (2006), 235-272. MR 2007i:34148 Zbl 1106.34061

[Bolibrukh 1990a] A. A. Bolibrukh, "Construction of a Fuchsian equation from a monodromy representation”, Mat. Zametki 48:5 (1990), 22-34, 158. MR 92d:14008 Zbl 0727.34005

[Bolibrukh 1990b] A. A. Bolibrukh, “The Riemann-Hilbert problem”, Uspekhi Mat. Nauk 45:2(272) (1990), 3-47, 240. In Russian; translated in Russ. Math. Surv. 45:2 (1990), 1-58. MR 92j:14014 Zbl 0706.34005

[Bott 1967] R. Bott, "A residue formula for holomorphic vector-fields", J. Differential Geometry 1 (1967), 311-330. MR 38 \#730 Zbl 0179.28801 
[Chudnovsky and Chudnovsky 1984] D. V. Chudnovsky and G. V. Chudnovsky, "Padé and rational approximations to systems of functions and their arithmetic applications", pp. 37-84 in Number theory (New York, 1982), edited by D. V. Chudnovsky et al., Lecture Notes in Math. 1052, Springer, Berlin, 1984. MR 86a:11029 Zbl 0536.10028

[Corel 2004] E. Corel, "On Fuchs' relation for linear differential systems", Compos. Math. 140:5 (2004), 1367-1398. MR 2005e:34270

[Deligne 1970] P. Deligne, Équations différentielles à points singuliers réguliers, Lecture Notes in Mathematics 163, Springer, Berlin, 1970. MR 54 \#5232 Zbl 0244.14004

[Fuchs 1866] L. I. Fuchs, "Zur Theorie der linearen Differentialgleichungen mit veränderlichen Coeffizienten”, J. Rein. Angew. Math. 66 (1866), 121-160.

[Gontsov 2004] R. R. Gontsov, "Improved Fuchs inequalities for systems of linear differential equations", Izv. Ross. Akad. Nauk Ser. Mat. 68:2 (2004), 39-52. In Russian; translated in Izv. Math. 68:2 (2004), 259-272. MR 2005b:34188 Zbl 1078.34069

[Gunning 1967] R. C. Gunning, Lectures on vector bundles over Riemann surfaces, University of Tokyo Press, 1967. MR 37 \#5888 Zbl 0163.31903

[Van Hoeij 1997] M. van Hoeij, "Formal solutions and factorization of differential operators with power series coefficients", J. Symbolic Comput. 24:1 (1997), 1-30. MR 98e:12006 Zbl 0924.12005

[Lando and Zvonkin 2004] S. K. Lando and A. K. Zvonkin, Graphs on surfaces and their applications, Encyclopaedia of Mathematical Sciences 141, Springer, Berlin, 2004. MR 2005b:14068 Zbl 1040.05001

[Levelt 1961] A. H. M. Levelt, "Hypergeometric functions, II", Nederl. Akad. Wetensch. Proc. Ser. A, 64 (1961), 373-385. MR 25 \#1302 Zbl 0124.03602

[Malgrange 1996] B. Malgrange, "Connexions méromorphes, II: Le réseau canonique", Invent. Math. 124:1-3 (1996), 367-387. MR 97h:32060 Zbl 0849.32003

[Van der Put and Saito 2009] M. van der Put and M.-H. Saito, "Moduli spaces for linear differential equations and the Painlevé equations", preprint, 2009. arXiv 0902.1702

[Van der Put and Singer 2003] M. van der Put and M. F. Singer, Galois theory of linear differential equations, Grundlehren der Mathematischen Wissenschaften 328, Springer, Berlin, 2003. MR 2004c:12010 Zbl 1036.12008

[Robba 1980] P. Robba, "Lemmes de Hensel pour les opérateurs différentiels: Application à la réduction formelle des équations différentielles”, Enseign. Math. (2) 26:3-4 (1980), 279-311 (1981). MR 82k:12022 Zbl 0466.12014

[Sabbah 2002] C. Sabbah, Déformations isomonodromiques et variétés de Frobenius, EDP Sciences, Les Ulis, Paris, 2002. MR 2003m:32013 Zbl 1101.14001

[Saito 1958] T. Saito, "On Fuchs' relation for the linear differential equation with algebraic coefficients”, Kōdai Math. Sem. Rep. 10 (1958), 101-104. MR 20 \#7124 Zbl 0086.06904

Received August 22, 2007. Revised July 20, 2009.

\section{EDUARDO COREL}

\section{GEORG-AUGUST UNIVERSITÄT GÖTTINGEN}

GOLDSCHMIDTSTR. 1

37077 GÖTTINGEN

GERMANY

ecorel@gwdg.de 